\title{
GENERALIZACIÓN DE LA ENTROPÍA DE KOLMOGOROV-SINAI: MAPAS Z-LOGÍSTICOS
}

\section{GENERALIZATION OF THE KOLMOGOROV-SINAI ENTROPY: Z-LOGISTIC MAPS}

\author{
${ }^{1}$ Garín F. Janampa A., ${ }^{2}$ Juan M. Pesantes R. y ${ }^{3}$ Martín B. Sandoval C.
}

\begin{abstract}
Resumen
En el umbral del caos, se calcula numéricamente la evolución temporal de la forma no extensiva de la entropía $S_{q}$ para los mapas z-logísticos. Se verifica la existencia de un único valor de $q \equiv q_{s e n} \leq 1$ para el cual la producción de entropía por unidad de tiempo de los mapas es finito. De esta manera se generaliza la entropía de Kolmogorv-Sinai.
\end{abstract}

Palabras clave: Caos, sistemas dinámicos, mapas z-logísticos, entropía.

\begin{abstract}
We numerically calculate, at the edge of chaos, the time evolution of the no extensive form of entropy $S_{q}$ for the z-logistic maps. We verify that only one value $q \equiv q_{\text {sen }} \leq 1$ exists such that the entropy production per unit time of these maps is finite, thus generalizing the Kolmogorv-Sinai entropy.
\end{abstract}

Key words: Chaos, Dynamical Systems, z-logistic maps, entropy.

\section{Introducción}

Una gran variedad de fenómenos naturales muestran un comportamiento complejo, impredecible y aparentemente caótico. Los especialistas en dinámica no lineal usan la palabra "caos" como un término técnico con un significado matemático preciso para definir un comportamiento irregular e impredecible de un sistema que es determinístico y no lineal, Hilborn R. C. (1994). Isaac Newton suponía que las ecuaciones de la mecánica clásica tenían que ver solo con un universo regular y ordenado, pero hoy en día se sabe que aquella suposición no es verdadera.

Tradicionalmente, los problemas fundamentales asociados con los orígenes del caos en flujos turbulentos venían siendo evitados, utilizando el argumento de que no sería humanamente posible describir estos fenómenos por la existencia de muchas partículas y grados de libertad. No es sorprendente que sistemas complicados, como la dinámica atmosférica o la variación del precio de una acción en la bolsa de valores, exhiban un comportamiento complejo, ya que la evolución de estos sistemas es influenciado por un número infinito de factores. Mientras tanto, se descubría que sistemas extremadamente simples podían exhibir un comportamiento caótico como los sistemas arriba mencionados.

En efecto, el proceso iterativo no lineal más simple posible, el mapa logístico estándar

$$
X_{t+1}=1-X_{t}^{2}, \quad\left(0<a \leq 2,-1 \leq X_{t} \leq 1(1)\right.
$$

exhibe ya un comportamiento dinámico complejo, bastante rico conforme varíe el valor del parámetro de control $a$, May R. (1976). Desde entonces innumerables trabajos de investigación se han desarrollado teniendo como modelo el mapa logístico y muchos otros mapas.

Los gráficos diseñados por la dinámica irregular de los mapas logísticos muestran un retrato del caos. Como muchos sistemas en la naturaleza, estos modelos matemáticos exhiben un comportamiento que parece ser aleatorio a pesar del hecho de que sus ecuaciones de movimiento son completamente determinados y al mismo tiempo los sistemas en cuestión son muy simples. De donde provienen los comportamientos complejos que estos sistemas simples muestran?

Desde el descubrimiento de la impredecibilidad en los sistemas deterministicos, los cuales conllevaron al estudio de sistemas dinámicos, muchos trabajos de investigación fueron desarrollados para encontrar las propiedades del caos con los cuales poder clasificar estos sistemas. Por ejemplo, indicadores dinámicos como la sensibilidad a las condiciones iniciales, los exponentes de Lyapunov, la entropía de Kolmogorv-Sinai (Pesin, Y. (1977), A. N. Kolmogorv (1958)) entre otros fueron desarrollados para hacer una caracterización más precisa de estos sistemas. Así por ejemplo, aparecen los denominados "sistemas-K" o sistemas de Kolmogorv que presentan un alto grado de irregularidad, suficiente para justificar una descripción estadística. Los sistemas-K exhiben una propiedad matemática conocida como "mezcla", es decir una entropía de Kolmogorv-Sinai positiva. Esto significa que los sistemas en cuestión son extremadamente sensibles a las condiciones iniciales, de modo que dos trayectorias inicialmente próximas divergen según una tasa exponencial. Esta tasa es medida por los exponentes de Lyapunov, que son equivalentes a la entropía de 
Kolmogorv-Sinai y pueden ser fácilmente calculados.

Entre tanto, recientemente se verifico la existencia de sistemas dinámicos naturales y artificiales para los cuales todos los indicadores conocidos no detectan la presencia del caos, pero los resultados numéricos muestran una elevada orden de impredecibilidad para las orbitas de estos sistemas. Este comportamiento fue denominado, dentro de la literatura científica, como caos "débil" para distinguirlo del caos "fuerte" (entropía de KolmogorvSinai positiva). El estudio de la dinámica del caos débil resulto ser importante para las aplicaciones prácticas. Hoy en día son conocidas las conexiones con muchos fenómenos físicos, biológicos y económicos: autoorganización critica, el umbral del caos, la dinámica intermitente, la difusión anómala y muchos otros. En estos ejemplos de la dinámica del caos débil, los indicadores estándar son nulos. Estos indicadores no pueden distinguir los comportamientos anómalos de los que tienen una dinámica trivial. Este problema condujo a la necesidad de generalizar estos indicadores dinámicos. Por esta razón, en la última década se han desarrollado estudios de fenómenos no lineales con nuevos conceptos y herramientas teóricas. Además cabe señalar la existencia de sistemas dinámicos caóticos no lineales que presentan un espacio de fase fractalico. Dos de los indicadores dinámicos más utilizados para caracterizar dichos sistemas son la sensibilidad a las condiciones iniciales (exponentes de Lyapunov) y la entropía de Kolmogorv-sinai, Pesin, Y. (1977), A. N. Kolmogorv (1958). Recientemente fueron introducidas las generalizaciones para estos indicadores inspirados en la entropía no extensiva, Tsallis, C., A. R. Plastino and W.-M Zheng (1997).

Una de las nuevas propuestas que promete una caracterización adecuada de estos sistemas dinámicos que presentan caos débil, está relacionada con la generalización no extensiva, Tsallis, J. (1988), de la estadística de Boltzmann-Gibbs, que en esta última década despertó mucho interés en las situaciones físicas en los cuales no se satisface las condiciones habituales de equilibrio (aleatoriedad insuficiente y movimiento limitados o movimiento no lineal en el espacio de fase pertinente), que conllevan a propiedades dinámicas anómalas Beck C., Benedek G., Rapisarda A. and Tsallis C. (2005), Beck C., Lewis G. S., and Swinney H. L. (2001), Campa A. Giansanti D. Moroni and C. Tsallis, (2001).

En 1988 C. Tsallis formulo una propuesta para generalizar la mecánica estadística de Boltzmann-Gibbs, basada en la forma entrópica:

$$
S_{q}(t)=K_{B} \frac{1-\sum_{i=1}^{W}\left[p_{i}(t)\right]^{q}}{q-1} \quad(q \in \mathcal{R})
$$

que restablece la entropía estándar de Boltzmann-Gibbs $S_{1}=-\sum_{i=1}^{W} p_{i} \ln p_{i}$ en el límite cuando $q \rightarrow 1$. Una de las características más importantes de esta nueva propuesta es la caracterización del índice entrópico $q$ el cual está relacionado con las propiedades intrínsecas de la dinámica microscópica del sistema. Una revisión general de las propiedades de la entropía no extensiva y sus aplicaciones, así como sus fundamentos teóricos pueden ser encontrados en: Brazilian Journal of Physics (1999), Murray Gell-Mann and Tsallis C, (2004).

El objetivo del presente artículo es la de caracterizar, dentro del marco de la mecánica estadística generalizada, sistemas dinámicos disipativos de baja dimensión (la familia de los mapas logísticos) para los dos casos, caos fuerte y caos débil (en el umbral del caos) por medio del indicador dinámico conocido como la entropía de Kolmogorv-Sinai.

\section{Materiales y métodos}

\section{Materiales}

Para un sistema dinámico caótico la entropía de Kolmogorov-Sinai (KS), se puede definir como el incremento por unidad de tiempo de la entropía estándar $S_{1}$ de Boltzmann-Gibbs (GB)

$$
S_{1}=-\sum_{i=1}^{W} p_{i} \ln p_{i} \cdot\left(K_{B} \equiv 1\right)(3)
$$

Por otro lado, la entropía KS está relacionada con los exponentes de Lyapunov por medio de la igualdad de Pesin, siempre en cuando los exponentes mencionados sean positivos, de lo contrario no es posible caracterizar los sistemas dinámicos a estudiarse. Para estos casos marginales en los que los exponentes de Lyapunov son nulos, tenemos bifurcaciones tangenciales y de doble periodo, así como el umbral del caos. Para estas situaciones anómalas se introdujo, Tsallis,C. A. R. Plastino and W.-M Zheng, (1997), una versión generalizada de la entropía KS, la cual se denotara con $K_{q}$, la misma que es definida como la tasa de incremento de la entropía no extensiva (ver formula 2):

$$
K_{q} \equiv \lim _{t \rightarrow \infty} \lim _{W \rightarrow \infty} \lim _{N \rightarrow \infty} \frac{<S_{q}>(t)}{t} \text { (4) }
$$

donde t es el tiempo, $\boldsymbol{W}$ es el numero de regiones del espacio de fase y $\boldsymbol{N}$ es el número de puntos (condiciones iniciales) que evolucionan con el tiempo.

\section{Metodologia.}

Considérese el mapa z-logístico (sistema dinámico)

$$
x_{t+1}=1-a\left|x_{t}\right|^{z},(5)
$$

d o n d e $1<z, 0<a \leq 2,-1 \leq x \leq 1$. E s t a familia de mapas presenta una ruta de doble periodo hacia el caos, mientras que el parámetro $z$ es la inflexión del mapa en su punto extremo. Los valores críticos del parámetro de dirección $a_{c}$ en el umbral del caos así como los valores correspondientes de $q$; los mismos que denotaremos por medio de $q_{\text {sen }}$, calculados según la fórmula (4) son mostrados en la tabla $\mathrm{N}^{\circ} 1$, para valores representativos de z. Todos los cálculos numéricos son 
desarrollados utilizando Fortran77, está claro que los mismos cálculos computacionales pueden ser realizados con MatLab, Matemática, etc.

Seguidamente se describe el procedimiento numérico desarrollado en esta investigación para calcular los índices entrópicos relevantes $q_{\text {sen }}(z)$. Este método fue introducido por vez primera en Tsallis C. (1998), para sistemas conservativos y utilizado en Latora V. and Baranger M. (1999) para el mapa logístico con $z=2$. Procedemos a dividir el intervalo-1 $\leq x \leq 1$ en $W$ ventanas iguales para el mapa z-logístico con un determinado valor del parámetro de dirección $a$. Posteriormente se elige (aleatoriamente o no) una de las ventanas y seleccionamos $N$ valores iniciales de $x$ todos en el interior de la ventana seleccionada previamente. A medida que t evoluciona, los $N$ puntos se desparraman dentro del intervalo del espacio de fase; de tal manera que obtenemos un conjunto $\left\{N_{i}(t)\right\}$ con

que conlleva a un conjunto de probabilidades

$$
\sum_{i=1}^{W} N_{i}(t)=N, \quad \forall t \quad\left\{p_{i}(t) \equiv \frac{N_{i}(t)}{N}\right\}
$$

Ya que para $t=0$ todos los $N$ puntos se encuentran dentro de la ventada escogida, $S_{q}(0)=0$ y a medida que el tiempo evoluciona se obtiene las curvas entrópicas $S_{q}(\mathbf{t})$.

Tabla 1. Mapas Z-Logísticos.

\begin{tabular}{ccc}
\hline$z$ & $a_{c}$ & $q_{\text {sen }}$ \\
\hline 1,10 & 1,1249885 & $-2,33 \pm 0,02$ \\
1,25 & 1,2095137 & $-0,78 \pm 0,01$ \\
1,50 & 1,2955099 & $-0,15 \pm 0,01$ \\
1,75 & 1,3550607 & $0,11 \pm 0,01$ \\
2,00 & 1,40115518909 & $0,24 \pm 0,01$ \\
2,50 & 1,4705500 & $0,39 \pm 0,01$ \\
3,00 & 1,5218787 & $0,47 \pm 0,01$ \\
\hline
\end{tabular}

\section{Resultados y discusión}

De los resultados obtenidos, utilizando la formula (4) para las curvas entrópicas $\boldsymbol{S}_{q}(\mathbf{t})$, puede observarse tres regiones sucesivas, Tsallis C, Mendes R. S. y Plastino A. R. (1998). En la primera región, la entropía es aproximadamente constante, por lo tanto no se puede obtener ninguna conclusión. En la segunda región, $\boldsymbol{S}_{q}(\mathbf{t})$ comienza a aumentar para que finalmente en la tercera región tienda asintóticamente hacia un valor de saturación. Dicho así, se espera que el aumento lineal de la entropía (con el valor $q \equiv q_{\text {sen }}$ ) se encuentre en la región intermedia.

En todos los casos, el crecimiento de $\boldsymbol{S}_{q}(\mathbf{t})$ en la región intermedia del tiempo es lineal cuando $q \equiv q_{\text {sen }}$, mientras que es cóncava para $q<q_{\text {sen }}$ y es convexa para $q>q_{\text {sen }}$, o sea, $\boldsymbol{K}_{q}$ es una magnitud finita para $q=q_{\text {sen }}$, diverge para $q<q_{\text {sen }}$ y es nula para $q>q_{\text {sen }}$
A fin de ofrecer una sustentación cuantitativa a este comportamiento, se ajusta las curvas entrópicas con el polinomio $S(t)=A+B t+C t^{2}$ en un intervalo $\left[t_{1}, t_{2}\right]$ caracterizando así la región intermedia. A continuación se define el coeficiente $R \equiv \frac{C\left(t_{1}+t_{2}\right)}{}$ como una medida de la importancia del término no $B$ lineal en el ajuste; así $\boldsymbol{R}$ es nulo para un ajuste estrictamente lineal. Los valores de los tiempos $t_{\mathbf{1}}$ y $t_{\mathbf{2}}$ son definidos como los extremos del región inicial, durante el cual $\boldsymbol{S}_{q}$ es aproximadamente constante, y el comienzo de la región de saturación respectivamente.

Está claro que $\boldsymbol{R}(\boldsymbol{q})$ es casi independiente de $\left(t_{\mathbf{1}}, t_{\mathbf{2}}\right)$ para cualquier conjunto de valores de específicos de $\left(t_{1}, t_{2}\right)$ que satisfagan las caracterizaciones arriba mencionadas.

\section{Conclusiones}

La entropía de Kolmogorv-Sinai $\boldsymbol{K}_{q}$ es un concepto importante para la caracterización de sistemas dinámicos caóticos disipativos (como los mapas analizados en este trabajo) y conservativos (como los Hamiltonianos clásicos de muchos cuerpos que satisfacen el teorema de Liouville). Su definición está basada en una división del espacio de fases accesible en un conjunto de $\boldsymbol{W}$ subespacios, que son visitados a lo largo del tiempo, con un cierto orden complejo que se origina de un punto único inicial en el espacio de fases. Con el método empleado, se escoge $N$ condiciones iniciales dentro de uno de los $\boldsymbol{W}$ subespacios, y a lo largo del tiempo estudiamos el conjunto de probabilidades $\left\{p_{i}\right\}(i=1,2,3, \ldots, W)$ asociadas con las ocupaciones de aquellos subespacios. El conjunto $\left\{p_{i}\right\}_{\text {permite el cálculo de la entropía }}$ $S_{1}\left(\left\{p_{i}\right\}\right)=-\sum_{i} p_{i} \mathbf{h} p_{i}$, esperando que $\boldsymbol{K}_{1}$ sea

el supremo del límite $\lim _{t \rightarrow \infty} \frac{S_{1}(t)}{t}$

El escenario descrito en este trabajo es solo uno los ya existentes, Acta Geophysica, V60, No 3 (2012). Sin embargo, es importante señalar que gracias a la formulación no extensiva de la entropía, este escenario nos permite caracterizar los indicadores dinámicos para el caso del umbral del caos, es decir para el denominado caos débil.

De esta manera se demostró numéricamente, que existe un único valor de $q_{s e n}$ tal que $K_{s e n}$ es finito, siendo igual a cero para $q>q_{s e n}$ e infinito para $q<q_{\text {sen }}$. $\mathrm{Si}$ el sistema dinámico no lineal fuese fuertemente caótico (mixtura exponencial en el espacio de fases), entonces $q_{\text {sen }}=1$, así obtenemos el escenario usual del caos fuerte. Pero, si el sistema fuese débilmente caótico (una mixtura del tipo de ley de potencias, o sea, con exponentes de Lyapunov nulos, pero con exponentes generalizados de Lyapunov positivos), entonces se espera que $q_{\text {sen }}<1$.

Como en el caso analizado en Latora V. and Baranger M. (1999), se verificó que el valor de $q_{\text {sen }}$ es precisamente el mismo obtenido previamente con dos procedimientos 
completamente diferentes, específicamente la sensibilidad a las condiciones iniciales según una ley de potencias, $\mathrm{y}$ la estructura multifractal del atractor caótico, Tsallis C., (2009). Esta unicidad del valor de $q_{\text {sen }}$ nos brinda una consistencia solida al escenario aquí descrito.

\section{Literatura citada}

Hilborn, RC. 1994, Chaos and Nonlinear Dynamics, Oxford Univ. Press, New York.

May R., Simple Mathematical Models with Very complicated Dynamics, Nature 261, 149 (1976).

Pesin, Y. 1977.Math. Surveys. 32, 55 (1977).

Kolmogorov, N. Doc. Acad. Nauk SSSR 119, 861 (1958).

Tsallis, C. A. R. P. and Zheng, W.-M.1997.Chaos, Solitons and Fractals 8, 885.

Tsallis, J. 1988. Stat. Phys. 52, 479-87

Beck, C.; Benedek, G.; Rapisarda, A. and Tsallis, C. C. 2005. Metastability and Nonextensivity, World Scientific, Singapore

Beck, C.; Lewis, GS. and Swinney, HL. 2001.Phys. Rev. E63, 35303

Campa, A.; Giansanti, D.; Moroni and C. Tsallis, 2001. Phys. Lett. A 286, 251

Brazilian Journal of Physics, 1999. Special Issue: Nonextensive Statistical Mechanics and Thermodynamics Vol. 29 - no 1 - March - (http://www.sbfisica.org.br/bjp/ ).

Gell-Mann, M. and Tsallis, C. 2004. Nonextensive Entropy, Oxford Univ. Press.

Buiatti, M.; Grigolino, P. and Palatella, L. 1999. Physica A268, 214.

Acta Geophysica, V60, No 3, 2012 "Statistical Mechanics in Earth Physics and Natural Hazards.

Latora V. and Baranger, M. 1999 .Phys. Rev. Lett. 82,520 .

Tsallis, C. 2009.Introduction to the Nonextensive Statistical Mechanics, Springer, New York 